Journal of Helminthology, Vol. II., No. 2, April, 1924, pp. 77-80.

\title{
On a new Cestode parasite of an East African Rock Rabbit (Procavia sp.).
}

\author{
By Jean G. Baer. \\ Zoological Department, University of Neuchatel (Switzerland).
}

INTRODUCTION.

Being engaged on a complete revision of the genus Inermicapsifer Janicki (1910), we had occasion to examine some material from the Helminthological Department of the London School of Tropical Medicine. This material was kindly placed at our disposal by Prof. R. T. Leiper, F.R.S., to whom we here tender our sincerest thanks. We are also indebted to Prof. O. Fuhrmann for his useful advice and criticisms.

The type material is deposited in the Helminthological Department of the London School of Tropical Medicine.

The material consists of two different lots, both, however, from $\mathrm{E}$. Africa, and is divided up as follows :-

\section{Parasite.}

Inermicapsifer hyracis (Rud.)

Inermicapsifer pagenstecheri (Setti)

Anoplocephala spatula (von Linstow)

Inermicapsifer lopas Bischoff

Inermicapsifer prionodes Bischoff

Inermicapsifer norhalli, $\mathrm{n}$. sp.
Locality.

Jequina.

Dodoma.

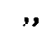

,

,

\section{Systematic Description. \\ ANOPLOCEPHALIDE, B1. 1848. \\ Linstowind, Fuhrm. 1907. \\ INERMICAPSIFER NORHALLI, n. sp.}

This new species is remarkable for its small size, the length being only $3.7-4.2 \mathrm{~mm}$. The greatest breadth of $0.95 \mathrm{~mm}$. is only attained in the last but one segment, the entire strobila consisting of 8-9 segments.

The scolex, $0.63 \mathrm{~mm}$. in diameter, is knob-shaped, without the least trace of any rostellar like structure, and is sharply separated from the 
rest of the strobila. The four suckers, $0.23 \mathrm{~mm}$. in diameter, are situated beneath the surface of the scolex, thus occupying the characteristic position of the genus Inermicapsifer. All the segments, except the last one, which is approximately square, are broader than long, the measurements being as follows :-1st segment, 0.2 to $0.5 \mathrm{~mm}$.; 2nd segment, 0.3 to $0.6 \mathrm{~mm}$.; 4 th segment, 0.4 to $0.7 \mathrm{~mm}$.; 6 th segment, 0.4 to $0.9 \mathrm{~mm}$.; 8th segment, 0.8 to $0.8 \mathrm{~mm}$.

On horizontal and transverse sections, we were only able to find the excretory ducts in the scolex and in the first segment, although the dorsal ducts are just visible in the second segment, their thicker wall being the better able to stand the increasing pressure of the growing genitalia.

The arrangement of the ducts is that typical of the genus, viz., the two dorsal ducts, $.006 \mathrm{~mm}$. in diameter, are situated slightly dorsally and outside the ventral ducts, $.011 \mathrm{~mm}$. in diameter, being connected with the latter by a network of exceedingly fine ducts.

The longitudinal muscles are arranged in two layers, one outer layer of isolated fibres, situated rather far apart in the parenchyma ; and one inner layer consisting of bundles of 3-4 fibres, regularly arranged, so that one bundle corresponds to about three or four fibres of the outer layer. The dorso-ventral muscles are well developed, whereas only a few odd fibres of transverse muscle are to be seen. Calcareous bodies are absent, both from the medullary and from the cortical parenchyma.

A feature shared in common with all adult Cestodes, having a restricted number of segments, is the extraordinary rapidity with which the genitalia appear. In the first segment we find the primordalia of the testes and of the male and female ducts; in the next segment the testes are already fully developed, as well as the cirrus pouch and vagina.

There are from 27-30 testes per segment, occupying the entire medullary parenchyma, and situated in one or two layers dorsal of the ovary. In transverse sections the testes appear as oval bodies, the long axis being dorso-ventral; they measure .11 to $.06 \mathrm{~mm}$.

In the fourth segment the testes begin to disappear to give way to the rapidly increasing ovary, and in the fifth segment only traces of these male gonads are to be seen.

The vas deferens is extremely convoluted, and leads into a large and powerful cirrus pouch, .25 mm. long and $.07 \mathrm{~mm}$. in diameter. The structure of this pouch is very interesting, being aberrant from the 
weakly developed type usually met with in this genus. The wall of the pouch is made up of six to seven layers of powerful circular muscles, there being only a single internal layer of longitudinal muscles; the cirrus is $.13 \mathrm{~mm}$. long and $.013 \mathrm{~mm}$. in diameter. An interesting feature, also found in other Cestodes, is the presence of a double layer of large transparent cells, surrounding the cirrus pouch, thus forming a sheath of "elastic" parenchyma. The presence of this tissue, as well as the whole structure of the cirrus pouch, seems to destine the latter to be able to withstand better the ever increasing pressure of the developing gonads. The position of the cirrus pouch and of the genital pore is characteristic for this species, the pouch being almost parallel to the edge of the segment, and the genital pore being situated in the posterior angle of the segment. The vagina, situated posteriorly to the cirrus pouch, is a fairly long thin walled tube, lined throughout with very fine hairs. In the fourth segment the greatest diameter is attained, as here the vagina is swelled with spermatozoa. The ovary appears as a definite structure in the fourth segment, and has already disappeared in the next. The small and compact vitelline gland is situated behind the ovary.

We have been unable to find any trace of a uterus, the ova appearing suddenly in the parenchyma in the fifth segment. Considering the fact that a uterus is found in all the other members of this genus, where the development of the gonads proceeds much more slowly, we do not hesitate to state that in the present species there is an ephemeral uterus.

In the sixth segment the ova are already grouped in clumps, surrounded by dark staining cells. The capsules are formed with their two layers of cells in the next segment, each capsule containing five to ten embryos, there being 18 to 25 large capsules, about $.2 \mathrm{~mm}$. in diameter. In the last two segments these capsules are situated mostly in two layers, being pushed on top of one another owing to lack of space.

The present species is the smallest adult member of this genus hitherto reported. It presents in size certain affinities with $I$. parvulus Bischoff (1913), and with I. lopas Bischoff (1913), but differs from the latter species in its anatomy, and also in having a much smaller number of segments, eight to nine, as compared to the 25 to 50 segments of the above mentioned species. 


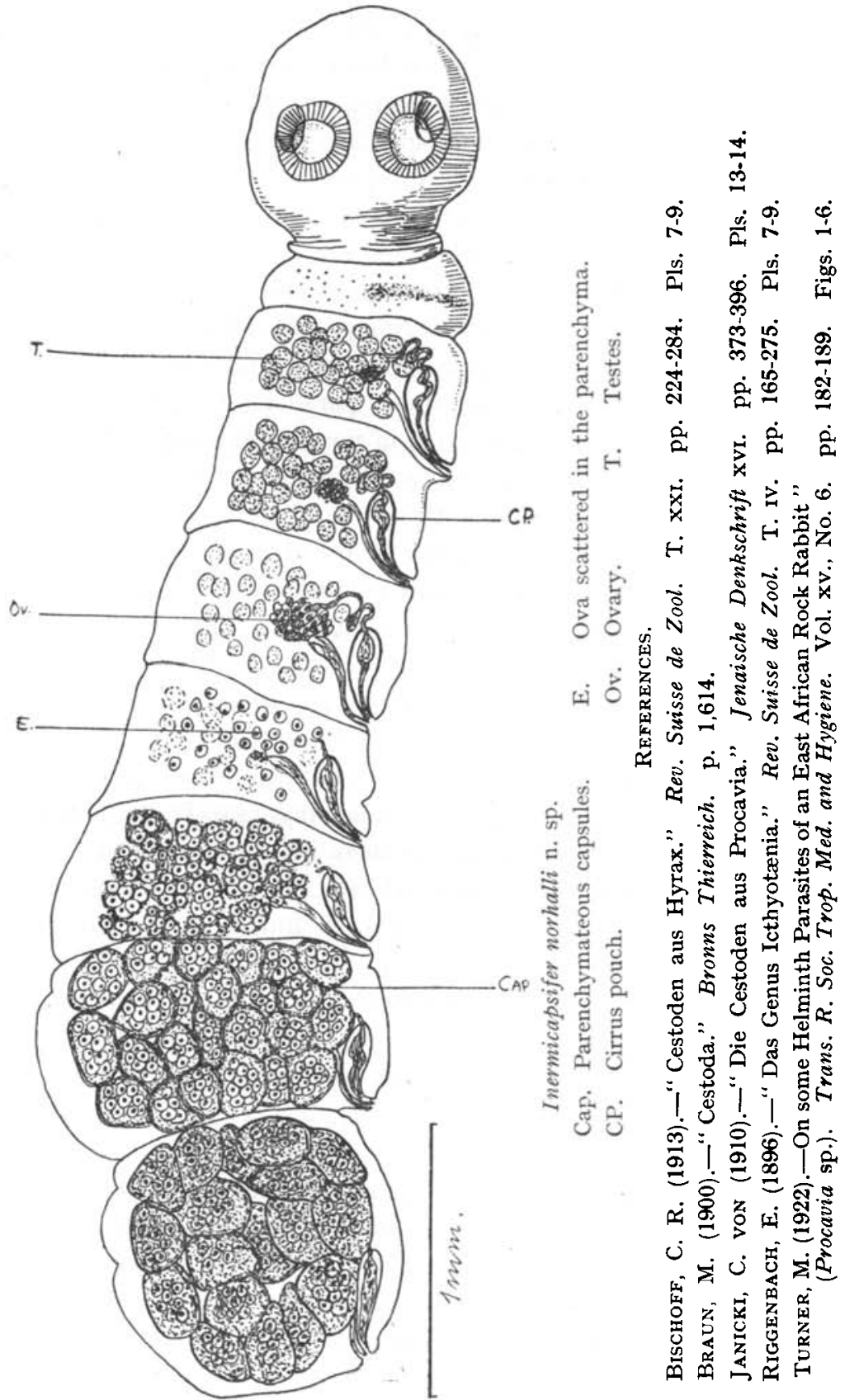

